Psychotherapeut 2010 - 55:5

DOI 10.1007/s00278-009-0718-y

Online publiziert: 17. Dezember 2009

(c) Springer-Verlag 2009

\title{
Wolfgang Schneider
}

Klinik und Poliklinik für Psychosomatik und Psychotherapeutische Medizin, Medizinische Fakultät, Universität Rostock

\section{Schwerpunktheft zum Thema Traumatherapeutische Konzepte}

Argument für diese Erwägungen wird angeführt, dass der Patient bis zum Eintreten der psychischen Symptomatik stets über gute Adaptationsfähigkeit verfügt und infolge der beruflichen Belastungssituation die typischen Symptome einer PTBS ausgebildet habe. Die Problematik der diagnostischen Kriterien für das Vorliegen einer PTBS oder einer anderen traumaassoziierten Störung wird durch diesen Fall exemplarisch verdeutlicht. Sind das Vorliegen einer längsschnittlich scheinbar unauffälligen Biografie und psychosozialen Anpassung sowie das Auftreten von für die PTBS beschriebenen charakteristischen Symptomen (z. B. Flashbacks, Albträume sowie innere und äußere Vermeidung) bereits ein genügender diagnostischer Beleg für die Gültigkeit der Diagnosenstellung? Wir finden im Alltag in den unterschiedlichsten sozialen und juristischen Kontexten - bei denen häufig Ansprüche auf Schadenersatz oder andere finanzielle Forderungen geprüft werden - oder auch im Asylrecht oder vor dem Hintergrund des Opferentschädigungsgesetzes vielfach klinische oder gutachterliche Problemstellungen, bei denen es um die Feststellung einer traumabedingten Störung geht. Vor diesem Hintergrund ist zukünftig eine Schärfung der diagnostischen Kriterien - die insbesondere das traumatische Ereignis betreffen sollten - zu wünschen. Von Interesse ist, welche diagnostischen Veränderungen die Entwicklungen des Diagnostic and Statistical Manual of Mental Disorders- (DSM-) $\mathrm{V}$ und der International Statistical Classification of Diseases and Related Health
Problems- (ICD-)11 zu diesem Themenkomplex bringen werden.

In zwei weiteren Arbeiten dieser Ausgabe steht das Thema Narzissmus im Mittelpunkt. E. Neumann untersucht empirisch die Konzepte des „offenen“ und „verdeckten“ Narzissmus und kann diese beiden Formen mit den eingesetzten Fragebogenmethoden an ihrer klinischen Stichprobe gut differenzieren.

Die Autoren C.-H. Lammers und M. Marwitz stellen das Konzept eines integrativen manualisierten Therapieansatzes zur Behandlung von Patienten mit einer narzisstischen Persönlichkeitsstörung vor. Neben stärker symptomorientierten Interventionen beinhaltet das Therapiekonzept auch beziehungs-, ressourcenund schemaorientierte Vorgehensweisen. Auch wenn dieser Ansatz primär an verhaltenstherapeutischen Konzepten orientiert ist, zeigt er doch relativ hohe Gemeinsamkeiten zu dem therapeutischen Modell von W. Wöller, das ebenfalls neben der Symptomfokussierung weiter gefasste psychologische Hintergrundvariablen der Erkrankung beinhaltet. Mit der Bezugnahme auf die Schematherapie öffnet sich das Konzept m. E. auch in Richtung psychodynamischer Entwicklungskonzepte, die der Schematherapie nicht fremd sein sollten.

\section{Korrespondenzadresse \\ Prof. Dr. Dr. Wolfgang Schneider}

Klinik und Poliklinik für Psychosomatik und Psychotherapeutische Medizin, Medizinische Fakultät, Universität Rostock

18147 Rostock

wolfgang.schneider@med.uni-rostock.de tionen zu einer Traumatisierung und einer nachfolgenden PTBS führen können. Als 